\title{
Novel FIB Torus Milling on Diamond Anvils with FIB Gasket Fabrication Enabling 5+ Megabar High Pressures Studies
}

\author{
N.E. Teslich ${ }^{1}$, Zs. Jenei ${ }^{1}$ and E.F. O’Bannon ${ }^{1}$ \\ 1. Lawrence Livermore National Laboratory, Livermore, CA, USA.
}

Today's Focused Ion Beam (FIB) microscopy capabilities provide essential techniques for microanalysis, site-specific TEM sample preparation, nano- and micro-machining for a wide range of materials and disciplines [1]. Static compression experiments are critical for developing physics and chemistry models that apply to many disciplines including modeling planetary interiors [2]. The diamond anvil cell (DAC) has been the primary tool for high pressure studies of materials up to $3 \mathrm{Mbar}$ however achieving higher pressures has been challenging and rare [3]. Recently FIB micro-machining has been used to mill a toroidal surface onto the central culet of the diamond anvil (fig.1b). FIB toroidal modification of a conventional single beveled diamond anvil (fig.1a) achieves higher pressures than unmodified single beveled diamond anvils [3]. Toroidal surfaces on the tip of the diamond anvils are designed to prevent gasket outflow and to provide confining pressure to the central culet area. Re gaskets are used between the diamond anvils to support the anvils and seal the sample in between the anvils. Re gaskets are pre-indented to a thickness of 5-10um (fig.2a) before FIB milling a 3-4um hole (fig.2b) for mounting the sample and pressure marker. When FIB milled diamond anvils are combined with FIB modified Re gaskets, high pressure capabilities have routinely produced over 5Mbar.

The single crystal diamond anvils are mounted on SEM stubs then C-coated and painted with colloidal graphite around the base up the facets not to include the flat surface, this is to reduce any potential charging or drift during milling times of $2+$ hours per diamond anvil. To obtain optimal shapes, the design is adapted to match each diamond based on SEM measurements taken from each individual diamond anvil prior to milling. We use these dimensions to generate the 3D diamond surface using our own codes, then we transform them into scaled 24bit bitmap images (fig.1c). These bitmaps are imported and used as the patterns for FIB milling. The milling currents are typically $13 \mathrm{nA}$ using a conventional $\mathrm{Ga}+$ ion beam employing a FEI Nova600i dual beam instrument. The process of FIB milling creates a 25-30nm amorphous layer that has not shown to significantly alter the achievable pressures. We are continuing to tune the parameters of torus shapes and gasket geometries. We have also FIB machine $\mathrm{W}$-discs that are as secondary gaskets (fig.2c). Further refinement of our toroidal design should make pressures of 7 Mbar achievable utilizing FIB milling with single-crystal toroidal diamond anvils [2].

References:

[1] N Teslich, GA Graham and JP Bradley, Microscopy Microanalysis (2007), p. 1534.

[2] Zs. Jenei et al., Nature Communications 9 (2018), p. 1.

[3] EF O’Bannon et al., Review of Scientific Instruments 89 (2018), p. 11

[4] This work was performed under the auspices of the US Department of Energy by Lawrence

Livermore National Laboratory under Contract No. DE-AC52-07NA27344 and was supported by the

LLNL-LDRD Program under Project No. 17-ERD-038 

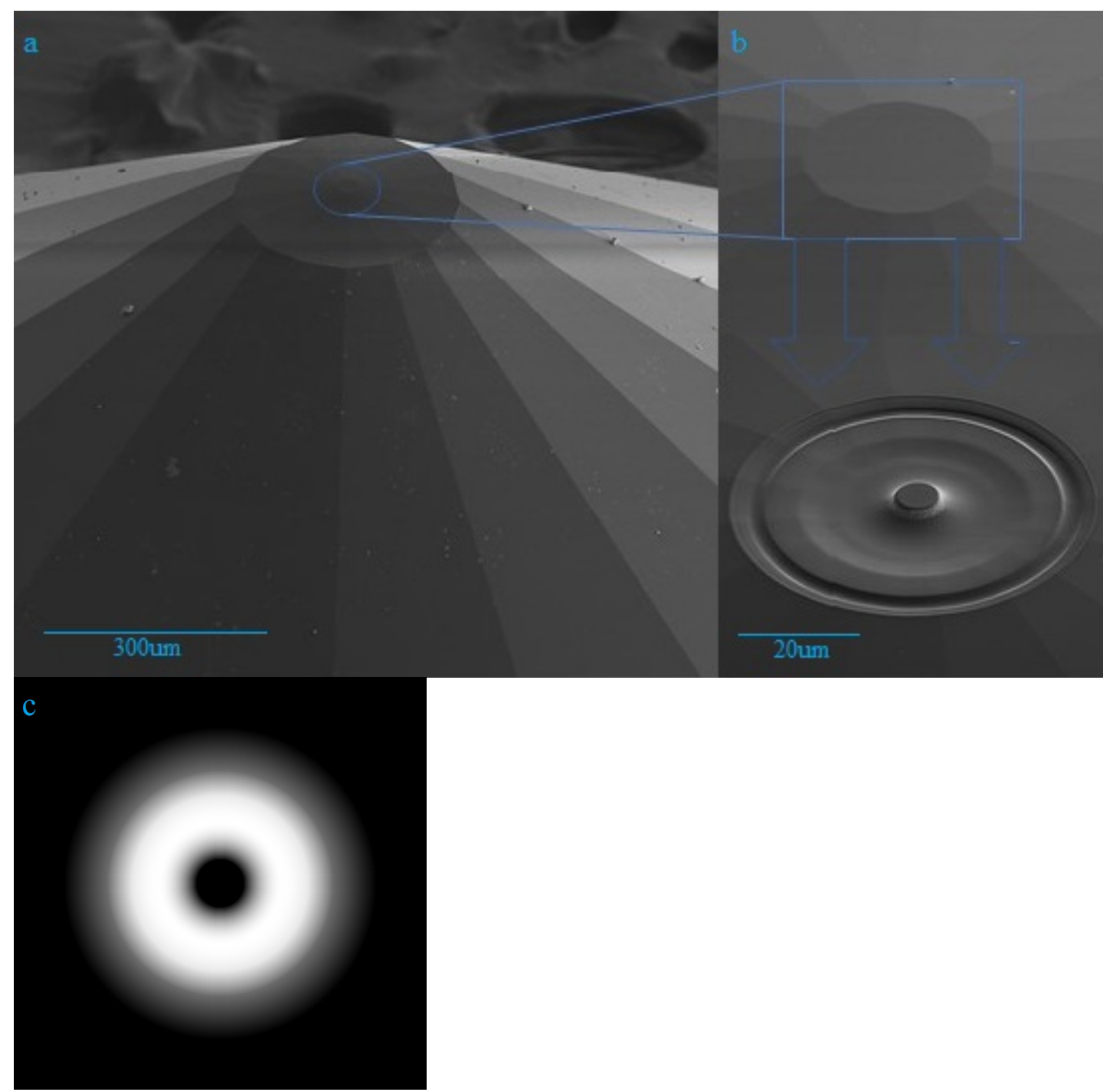

Figure 1. a) Conventional (DAC) single beveled 30um diamond flat. b) FIB milled torus. c) Custom 3D 24bit bitmap generated for FIB milling.

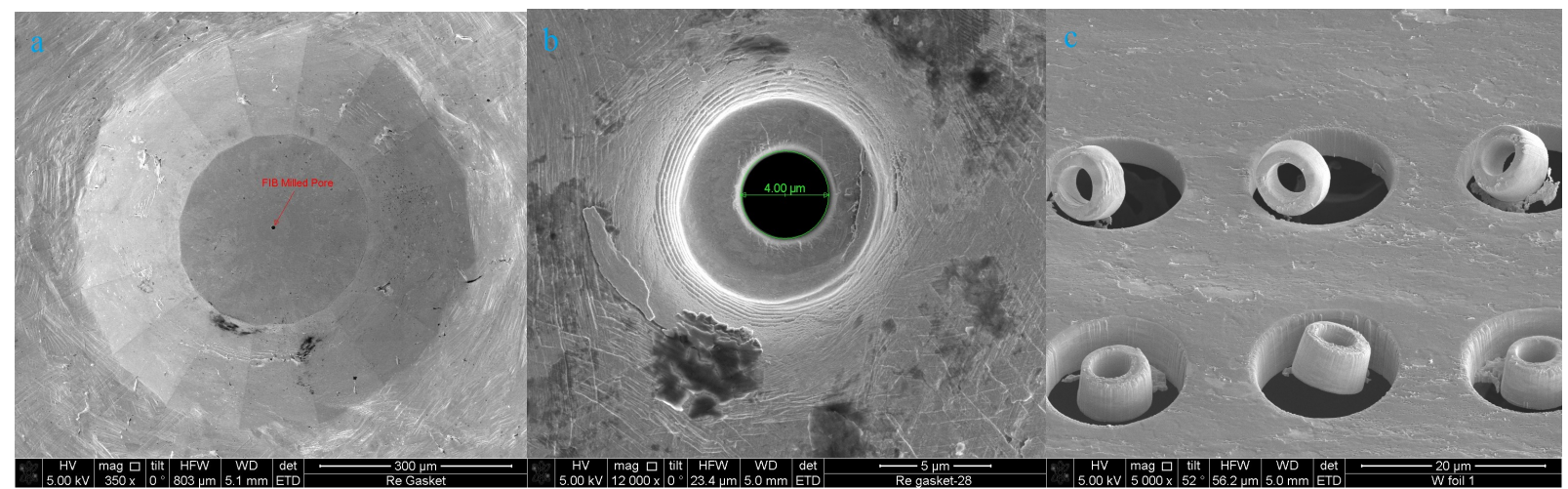

Figure 2. a) Pre-indented Re gasket. b) FIB milled 4um pore in gasket. c) FIB milled W-disks 